\title{
Radiobiological evaluation of the influence of dwell time modulation restriction in HIPO optimized HDR prostate brachytherapy implants
}

\author{
Panayiotis Mavroidis, PhD', Zaira Katsilieri, MSc², Vasiliki Kefala, MSc², Natasa Milickovic, PhD², \\ Nikos Papanikolaou, PhD³, Andreas Karabis, PhD4, Nikolaos Zamboglou, MD, PhD5, Dimos Baltas, PhD2,6 \\ IDepartment of Medical Radiation Physics, Karolinska Institutet and Stockholm University, Sweden, ${ }^{2}$ Department of Medical \\ Physics \& Engineering, Strahlenklinik, Klinikum Offenbach GmbH, Germany, ${ }^{3}$ Department of Radiological Sciences, University of Texas \\ Health Sciences Center, San Antonio, Texas, USA, 4Pi-Medical Ltd., Athens, Greece, 5 Strahlenklinik, Klinikum Offenbach GmbH, Germany, \\ 6Nuclear and Particle Physics Section, Physics Department, University of Athens, Greece
}

\begin{abstract}
Purpose: One of the issues that a planner is often facing in HDR brachytherapy is the selective existence of high dose volumes around some few dominating dwell positions. If there is no information available about its necessity (e.g. location of a GTV), then it is reasonable to investigate whether this can be avoided. This effect can be eliminated by limiting the free modulation of the dwell times. HIPO, an inverse treatment plan optimization algorithm, offers this option. In treatment plan optimization there are various methods that try to regularize the variation of dose non-uniformity using purely dosimetric measures. However, although these methods can help in finding a good dose distribution they do not provide any information regarding the expected treatment outcome as described by radiobiology based indices.

Material and methods: The quality of 12 clinical HDR brachytherapy implants for prostate utilizing HIPO and modulation restriction (MR) has been compared to alternative plans with HIPO and free modulation (without MR). All common dose-volume indices for the prostate and the organs at risk have been considered together with radiobiological measures. The clinical effectiveness of the different dose distributions was investigated by calculating the response probabilities of the tumors and organs-at-risk (OARs) involved in these prostate cancer cases. The radiobiological models used are the Poisson and the relative seriality models. Furthermore, the complication-free tumor control probability, $P_{+}$and the biologically effective uniform dose $(\overline{\bar{D}})$ were used for treatment plan evaluation and comparison.

Results: Our results demonstrate that HIPO with a modulation restriction value of 0.1-0.2 delivers high quality plans which are practically equivalent to those achieved with free modulation regarding the clinically used dosimetric indices. In the comparison, many of the dosimetric and radiobiological indices showed significantly different results. The modulation restricted clinical plans demonstrated a lower total dwell time by a mean of $1.4 \%$ that was proved to be statistically significant $(p=0.002)$. The HIPO with MR treatment plans produced a higher $P_{+}$by $0.5 \%$, which stemmed from a better sparing of the OARs by $1.0 \%$.

Conclusions: Both the dosimetric and radiobiological comparison shows that the modulation restricted optimization gives on average similar results with the optimization without modulation restriction in the examined clinical cases. Concluding, based on our results, it appears that the applied dwell time regularization technique is expected to introduce a minor improvement in the effectiveness of the optimized HDR dose distributions.
\end{abstract}

Key words: HIPO, HDR, optimization, radiobiology, treatment plan.

\section{Purpose}

For HDR brachytherapy, inverse planning should result in a faster and reproducible planning process, producing high quality treatment plans that closely match the clinical protocol constraints [1,2]. Especially for the treatment of prostate cancer, a number of algorithms have been proposed during the last decade based on quasi-Newton, Linear Programming, Simulated Annealing and heuristics [3-7]. Many of them have been implemented in modern Treatment Planning Systems (TPS) and are in clinical use for years, and they are considered as a mature technology $[8,9]$.

Modern inverse optimization technology enables a very fast adjustment of the source dwell time distribution within 
implanted catheters according to user-defined objectives and penalties for the target volume(s) and organs at risk (OARs) in HDR brachytherapy. This adjustment generally does not account for any smoothness criterion for the variation of dwell times within catheters. It is a common characteristic for HDR implants optimized with such algorithms that there are a few very dominating dwell positions where the largest part of the total dwell time is spent [10]. This obviously leads to a selective extension of high doses in volumes around such dwell positions. If there is no information available about its necessity (e.g. to increase the dose at the location of a GTV), then it is reasonable to investigate whether this can be avoided [11].

In the present work, the TPS Oncentra Prostate v.3.0 (Nucletron B.V., Veenendaal, The Netherlands) was used. Oncentra Prostate has implemented the Hybrid Inverse treatment Planning and Optimization (HIPO) algorithm $[4,12]$. HIPO is a 3D anatomy-based inverse planning algorithm which is not only capable of optimizing the dose distribution for a given needle configuration but also capable of finding an adequate needle configuration for each application. Furthermore, HIPO offers a modulation restriction option that limits the free modulation of dwell times according to a user-selectable level, thus eliminating the selective hot spots.

The present work is based on the 3D ultrasound (U/S) image sets and clinical treatment plans of 12 patients obtained intraoperatively right after the needle implantation (the clinical plans are based on these 3D-U/S image sets). All the inverse optimized treatment plans were based on HIPO using the modulation restriction methods, which are investigated in conjunction with radiobiological dose non-uniformity evaluation measures in order to estimate their expected clinical impact [13]. A certain pattern of dose non-uniformities produces a certain radiobiological response. However, most of the existing radiobiological models do not maintain the information of the spatial distribution. Consequently, different dose distributions producing the same pattern of DVHs would result in the same radiobiological response even though they are characterized by different patterns of dose nonuniformities.

The low tolerance doses, which characterize the involved organs-at-risk (OAR) are usually the major constraints in brachytherapy. The dose delivered to the tumors is further limited when these OARs are in close proximity to the gross tumor volume (GTV) and clinical target volume (CTV). The selection of the best treatment plan can be a challenging task due to variations in the optimization methodology. Isodose charts, dose volume histograms (DVH), dose-volume parameters and conformity based indices such as COIN are currently used for treatment plan evaluation $[14,15]$. However, all these evaluation measures are only dose based and they do not take the radiobiological characteristics of tumors or normal tissues into account. Consequently, radiobiological measures have also to be employed in order to estimate the response of the different tissues and the overall expected treatment outcome. In this analysis, the use of the biologically effective uniform dose $(\overline{\bar{D}})$ and complicationfree tumor control probability $\left(P_{+}\right)$have been applied as treatment plan evaluation tools $[16,17]$. By applying these radiobiological measures, the effectiveness of the treatment plans, which were developed with and without the application of modulation restriction, are investigated for the 12 prostate cancer cases.

\section{Material and methods}

A certain modulation restriction and treatment plan optimization method was applied to the examined prostate HDR treatment plans. The critical structures included the urethra, bladder and rectum. The 3D-U/S based preplanning, the transperineal implantation of needles using template and the 3D-U/S based intraoperative planning and irradiation were realized using the real-time dynamic planning system Oncentra Prostate version 3.0 and the MicroSelectron HDR Vs. 3.0 Afterloader (both Nucletron B.V., The Netherlands).

\section{DVH based parameters in brachytherapy}

For the evaluation and documentation of the dose distributions, GEC/ESTRO-EAU proposed DVH-based parameters, which are described below [18-21], have been considered.

\section{PTV - Oriented parameters}

$D_{100}$ : The dose that covers $100 \%$ of the PTV volume, which is the strict definition of the Minimum Target Dose (MTD).

$D_{90}$ : The dose that covers $90 \%$ of the PTV volume. A $D_{90}$ value of greater than or equal to the prescription dose is a measure of a good implant quality.

$V_{100}$ : The percentage of prostate volume (PTV) that has received at least the prescription dose $(100 \%=$ prescribed dose).

$V_{150}$ : The volume that has received $50 \%$ more than the prescribed dose (150\% of the prescription dose).

\section{OAR - Oriented parameters}

The dose should be related to fixed points and/or fixed volumes, even if there is no general agreement on certain points or fixed volumes at present [21-26]. There are suggestions to use the maximum doses for the OARs, where the maximum doses are considered to be $[18,21]$ :

$D_{2 \mathrm{~cm}^{3}}$ : the dose for the most exposed $2 \mathrm{~cm}^{3}$ of rectum or bladder,

$D_{0.1 \mathrm{~cm} 3}$ : the dose for the most exposed $0.1 \mathrm{~cm}^{3}$ of the urethra as the best estimation of the maximum dose,

$D_{10}$ : the highest dose covering $10 \%$ of the OAR volume (rectum, bladder, urethra)

\section{The conformal index COIN}

In Baltas et al. [14] a conformal index COIN was proposed as a measure of implant quality and dose specification in brachytherapy. COIN focusing on the target volume PTV is defined as:

$$
\operatorname{COIN}=c_{1} \cdot c_{2}
$$

The coefficient $c_{1}$ is the fraction of the PTV that is enclosed by the prescription dose. The coefficient $c_{2}$ is the fraction of the volume encompassed by the prescription 
dose that is covered by PTV. It is a measure of how much tissue outside the PTV is covered by the prescription dose. COIN has been extended to consider also the conformity of the 3D dose distribution regarding the OARs $[14,15]$ :

$$
\mathrm{COIN}=c_{1} \cdot c_{2} \cdot c_{3}
$$

where

$$
c_{3}=\prod_{i=1}^{N_{\text {OAR }}}\left[1-\frac{\mathrm{V}_{\text {OAR }}^{i}\left(D>D_{\text {limit }}^{i}\right)}{\mathrm{V}_{\text {OAR }}^{i}}\right]
$$

where $N_{\mathrm{OAR}}$ is the total number of OARs, $V_{\mathrm{OAR}}^{i}$ is the volume of the $i$-th OAR, $D_{\text {limit }}^{i}$ is the dose limit defined for the $\mathrm{i}$-th OAR and $V_{\mathrm{OAR}}^{i}\left(D>D_{\text {limit }}^{i}\right)$ is the volume of the $\mathrm{i}$-th OAR that receives a dose that exceeds the dose limit $D_{\text {limit. }}^{i}$

The ideal situation is COIN $=c_{1}=c_{2}=c_{3}=1$. COIN assumes in this form that the PTV, the OARs and the surrounding NT are of the same importance.

\section{Clinical material}

Twelve clinical implants for HDR brachytherapy of prostate cancer were selected as monotherapy for low-risk cases out of the patient database in Strahlenklinik Offenbach. These twelve implants cover the whole range of prostate volumes with a full range of $26-101 \mathrm{~cm}^{3}$. A summary of characteristics of the clinical protocol applied on the twelve implants is listed in the Table 1. The clinical procedure is totally $3 \mathrm{D}$ ultrasound based using the real time intraoperative planning system Oncentra Prostate version 3.0 (OcP, Fa.Nucletron B.V., The Netherlands) and MicroSelectron HDR version 3 Genius. Inverse planning was based on HIPO algorithm, which has been implemented in Oncentra Prostate and supports: (a) inverse optimization of dwell times for a given needle configuration and (b) inverse optimization of needle positions and dwell times. In the present study, only the clinically placed needles were used for inverse planning
(Table 2). It was based on dosimetric objectives, which are linearly penalizing over/under dosage in target(s) while protecting OARs from overdosage [4]. Furthermore, in order to get restriction of the free modulation of dwell times allowing thus more smooth source movements and more smooth distributions of dwell time over dwell positions, HIPO offers the option of a modulation restriction (MR) parameter, the dwell time gradient restriction (DTGR). It considers the gradient of the dwell times of the source within the separate catheters in the form of a dedicated objective function. The MR parameter takes values in the range $[0.0,1.0]$. A value of 0.0 will make the system ignore this dwell time gradient objective, whereas a value of 1.0 results in the maximum consideration of it. In Strahlenklinik Offenbach, the HDR Monotherapy is delivered in three implants separated by at least 2 weeks interval. In each implant a single fraction with a prescription dose of $11.5 \mathrm{~Gy}$ was delivered thus resulting in a total brachytherapy dose of $34.5 \mathrm{~Gy}$. The prostate gland $\left(\mathrm{CTV}_{1}\right)$ was considered as PTV and urethra, bladder and rectum are used as OARs in the treatment planning.

Table 1. Clinical protocol in Offenbach Clinic for HDR monotherapy

\begin{tabular}{lcc} 
& \multicolumn{1}{c}{ Parameter } & Value \\
\hline \multirow{3}{*}{ Prostate } & Reference dose & $11.5 \mathrm{~Gy}(=100 \%)$ \\
\cline { 2 - 3 } & $\mathrm{D}_{90} 0^{- \text {Prostate }}$ & $\geq 100 \%(=11.5 \mathrm{~Gy})$ \\
\cline { 2 - 3 } & $\mathrm{V}_{100^{-} \text {-Prostate }}$ & $\geq 90 \%$ \\
\cline { 2 - 3 } & $\mathrm{V}_{150^{-} \text {-Prostate }}$ & $\leq 35 \%$ \\
\hline Urethra & $\mathrm{D}_{10}$-Urethra & $\leq 115 \%(=13.2 \mathrm{~Gy})$ \\
\cline { 2 - 3 } & $\mathrm{D}_{0.1 \mathrm{~cm}^{3} \text {-Urethra }}$ & $\leq 120 \%(=13.8 \mathrm{~Gy})$ \\
\hline Rectum & $\mathrm{D}_{10}$-Rectum & $\leq 75 \%(=8.6 \mathrm{~Gy})$ \\
\cline { 2 - 3 } & $\mathrm{D}_{0.1 \mathrm{~cm}^{3} \text {-Rectum }}$ & $\leq 80 \%(=9.2 \mathrm{~Gy})$ \\
\hline Bladder & $\mathrm{D}_{10}$-Bladder & $\leq 75 \%(=8.6 \mathrm{~Gy})$ \\
\cline { 2 - 3 } & $\mathrm{D}_{0.1 \mathrm{~cm}^{3} \text {-Bladder }}$ & $\leq 80 \%(=9.2 \mathrm{~Gy})$
\end{tabular}

Table 2. Summary of the characteristics of the 12 clinical implants of HDR brachytherapy as monotherapy for

\begin{tabular}{|c|c|c|c|c|c|c|c|}
\hline $\begin{array}{l}\text { Case } \\
\text { No. }\end{array}$ & $\begin{array}{c}\left(P T V=C T V_{1}\right) \\
\left(\mathrm{cm}^{3}\right)\end{array}$ & No catheters & Catheter type & $\begin{array}{l}\text { Source step } \\
(\mathrm{mm})\end{array}$ & No ASDPs & $\begin{array}{l}\text { ASDPs } \\
\text { per } \mathrm{cm}^{3}\end{array}$ & MR values \\
\hline 1 & 26 & 16 & metallic & 2.5 & 217 & 8.3 & 0.15 \\
\hline 2 & 27 & 14 & plastic & 2.5 & 183 & 6.8 & 0.20 \\
\hline 3 & 36 & 14 & metallic & 2.5 & 197 & 5.5 & 0.10 \\
\hline 4 & 36 & 15 & plastic & 2.5 & 195 & 5.4 & 0.20 \\
\hline 5 & 38 & 14 & plastic & 2.5 & 188 & 4.9 & 0.10 \\
\hline 6 & 42 & 15 & metallic & 2.5 & 209 & 5.0 & 0.15 \\
\hline 7 & 48 & 14 & plastic & 2.5 & 239 & 5.0 & 0.12 \\
\hline 8 & 63 & 16 & metallic & 2.5 & 259 & 4.1 & 0.15 \\
\hline 9 & 64 & 18 & plastic & 2.5 & 309 & 4.8 & 0.12 \\
\hline 10 & 76 & 15 & plastic & 2.5 & 283 & 3.7 & 0.10 \\
\hline 11 & 80 & 18 & metallic & 2.5 & 313 & 3.9 & 0.10 \\
\hline 12 & 101 & 18 & plastic & 2.5 & 352 & 3.5 & 0.20 \\
\hline
\end{tabular}
low risk prostate cancer 

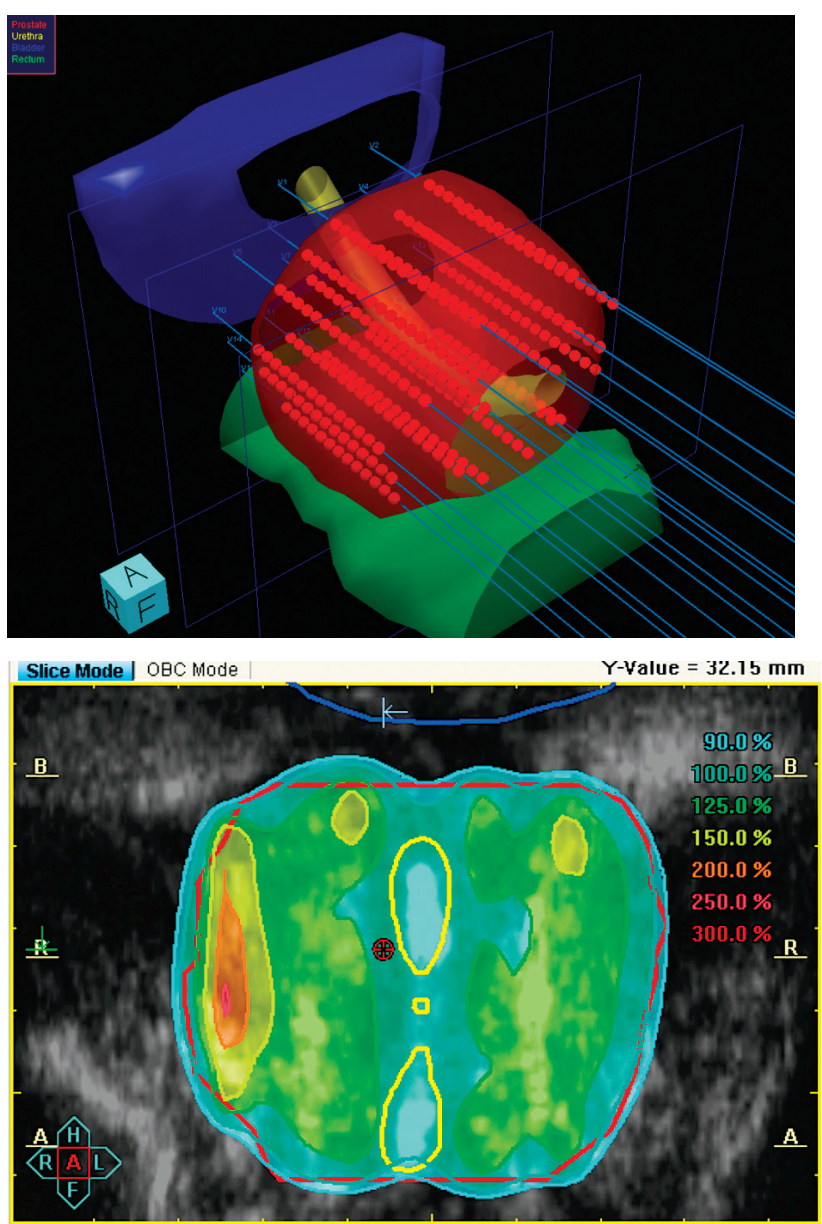

Slice Mode | OBC Mode

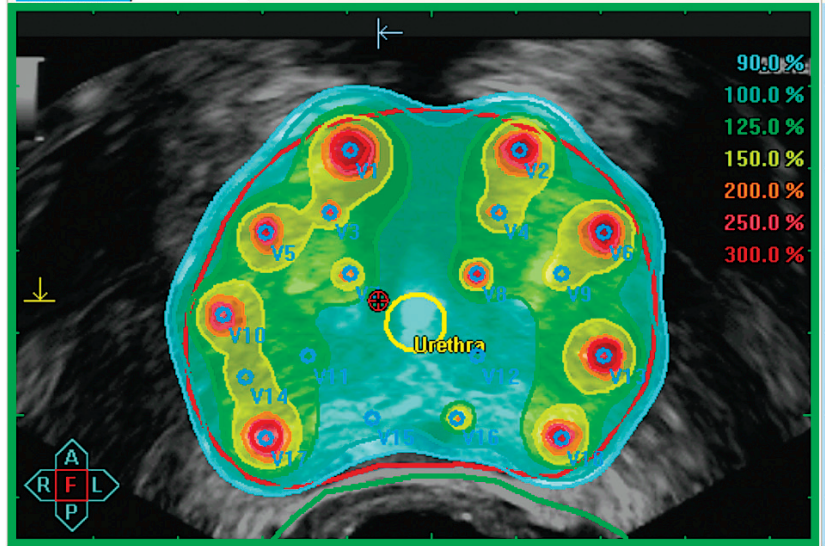

The whole procedure including dose delivery was realized intraoperatively utilizing 3D and 2D Ultrasound imaging.

For each of these twelve patients the clinically used implant was defined as the reference plan. All the clinical implants were inversely planned using HIPO with modulation restriction (MR). The optimization settings consisted of a maximum and/or minimum dose to a VOI and its importance factor (penalty) as well as the MR parameter. During the clinical procedure, the MR values were selected based on the maximum values resulting in plans that completely fulfilled the constraints of the dosimetric protocol. For each of these twelve implants an
Fig. 1. With the help of the OcP software, virtual catheters are created (blue catheters). This can be done either automatically (HIPO utilizing dosimetric objective functions) or manually by the user. The resulting 3D dose distribution fulfills the clinical protocol

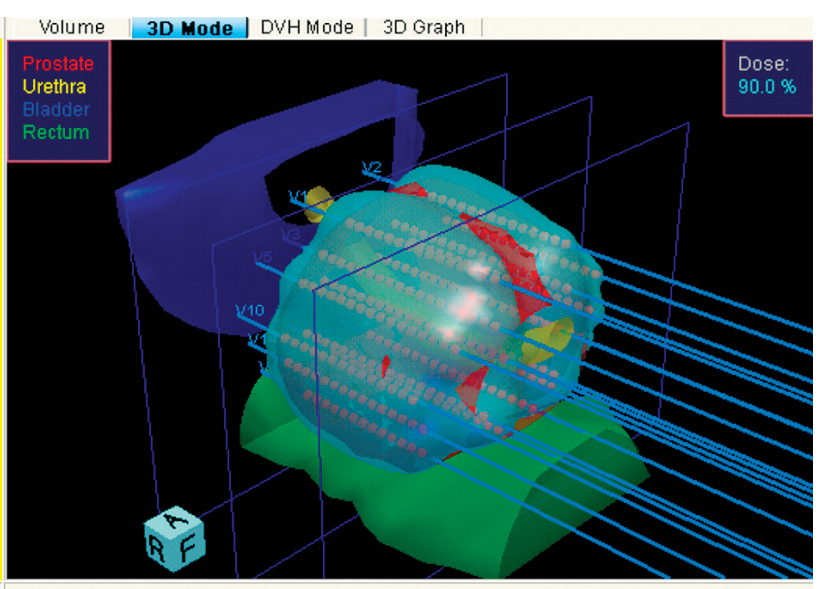

Slice Mode | OBC Mode

$x$-value $=45.77 \mathrm{~mm}$

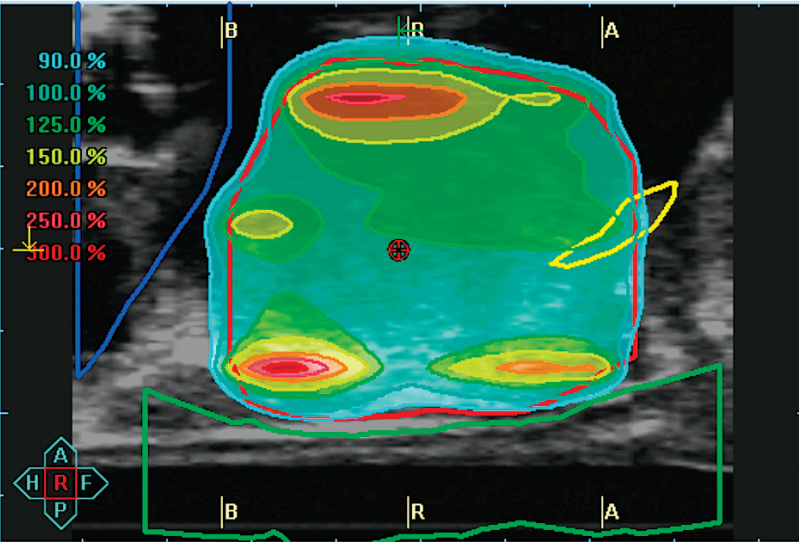

additional plan without modulation restriction $(\mathrm{MR}=0)$ was produced for the purpose of this project. In the treatment plan evaluation, the individual tissue DVHs of both plans, with (clinical) and without $(\mathrm{MR}=0$, theoretical) modulation restriction, were calculated. All the relevant dose-volume parameters, for the prostate and OARs (rectum, urethra and bladder) were considered as dosimetric indices with the inclusion of all the internationally recommended values.

In Fig. 1, the HIPO optimized catheter and dwell position configuration for one of the patients is presented. Based on the defined Volumes of Interest (VOIs) and the 
preset values, the several objectives regarding PTV, GTV, Normal Tissue stroma (NT) and OARs can be activated or deactivated and the corresponding low and high dose limit values and importance factors (penalties) can be accepted or changed. NT is considered to be a thin layer of tissue surrounding the PTV where no other VOIs are defined by the user. NT is utilized for avoiding the uncontrolled expansion of the dose outside the PTV, in areas where no OARs are defined. HIPO supports a protocol based planning, allowing thus the definition and storage of several sets of objectives, dose limits, importance factors and prescription doses for dealing with different clinical protocols e.g. for monotherapy, boost or salvage treatments.

An example of the impact of the Modulation Restriction (MR) algorithm is shown in Fig. 2, where the corresponding dwell times are demonstrated together with the individual tissue DVHs for the two MR values examined. In this Figure, the significant change of dwell times in several dwell positions can be observed. In the dwell positions, where the dwell time was extremely high, a redistribution of time among neighbors of that source dwell position is resulted when the modulation restriction module is activated. The upper diagram of Fig. 2 demonstrates a graphical comparison of the dwell time profiles of the two plan categories for a prostate cancer case. There was a total dwell time reduction when the Modulation Restriction (MR $=0.12$ ) is used. Despite the significant differences in the dwell time distribution within catheters no significant changes in the DVHs of the prostate and OARs were observed (lower diagram of Fig. 2).

\section{Radiobiological measures for treatment plan evaluation}

The response of a normal tissue to a non-uniform dose distribution is given by the relative seriality model, which accounts for the volume effect. For a heterogeneous dose distribution, the overall probability of injury $P_{\mathrm{I}}$, for a number of OARs is expressed as follows $[27,28]$ :

$P_{\mathrm{I}}=1-\prod_{j=1}^{N_{\text {organs }}}\left(1-P_{\mathrm{I}}^{j}\right)=1-\prod_{j=1}^{N_{\text {organs }}}\left(1-\left[1-\prod_{i=1}^{\mathrm{M}_{j}}\left(1-\mathrm{P}^{j}\left(\mathrm{D}_{i}\right)^{S_{j}}\right)^{\Delta v_{i}}\right]^{1 / S_{j}}\right)$

where $P_{\mathrm{I}}^{j}$ is the probability of injuring organ $j$ and $\mathrm{N}_{\text {organs }}$ is the total number of OARs. $\mathrm{P}^{j}\left(D_{i}\right)$ is the probability of response of the organ $j$ having the reference volume and
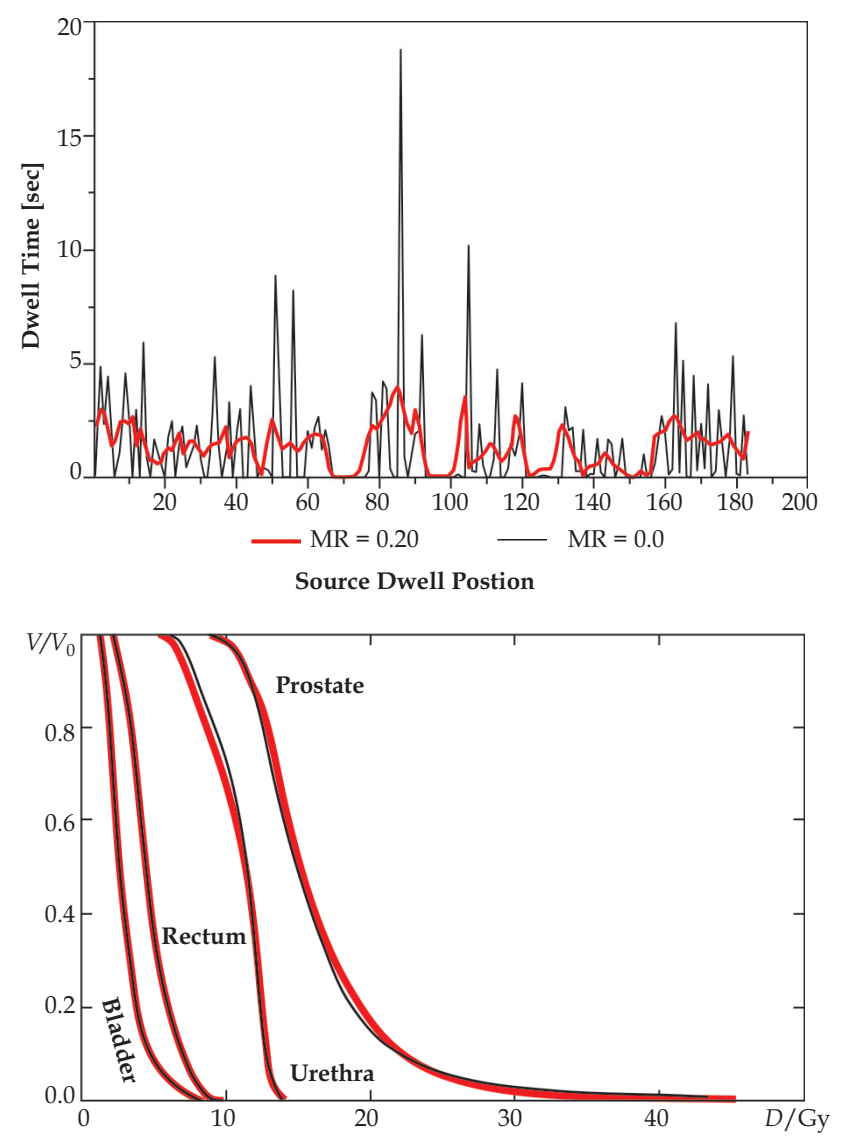

Fig. 2. Upper diagram: Comparison of the dwell time profiles for the two types of modulation ( $M R=0.20$ and $M R=0$ ) for the case 2 in Table 2. Lower diagram: Comparison of the DVHs for prostate, urethra, bladder and rectum for the two types of modulation ( $\mathrm{MR}=0.20$ [red thick line] and $\mathrm{MR}=0$ [black thin]) is shown for the same case. The prescription dose of the fraction is $11.5 \mathrm{~Gy}(100 \%)$

being irradiated to dose $\mathrm{D}_{i} . \Delta_{v i}=\Delta V_{i} / V_{\text {ref }}$ is the fractional subvolume of the organ $\left(\Delta V_{i}\right)$ that is irradiated at the dose level $D_{\mathrm{i}}$ compared to the reference volume $\left(V_{\text {ref }}\right)$ for which the values of the model parameters were calculated. $M_{j}$ is the total number of voxels or subvolumes in the organ $j$, and $s_{j}$ is the relative seriality parameter that characterizes the internal organization of that organ. A relative seriality close to zero $(s \approx 0)$ corresponds to a completely parallel structure, which becomes non-functional when all its

Table 3. Summary of the model parameter values for the prostate cancer cases. $D_{50}$ is the $50 \%$ response dose, $\gamma$ is the maximum normalized value of the dose-response gradient and $\mathrm{s}$ is the relative seriality, which characterizes the volume dependence of the organ

\begin{tabular}{lccccc} 
Radiobiological model & $\boldsymbol{D}_{50}(\mathrm{~Gy})$ & $\boldsymbol{\gamma}$ & $\boldsymbol{s}$ & $\boldsymbol{\alpha} / \boldsymbol{\beta}$ & Endpoint \\
\hline PTV & 70.0 & 4.0 & - & 3.0 & control \\
\hline Urethra (U) & 120.0 & 3.0 & 0.03 & 3.0 & stenosis \\
\hline Bladder (B) & 80.0 & 3.0 & 0.3 & 3.0 & $\begin{array}{c}\text { symptomatic contracture, } \\
\text { volume loss }\end{array}$ \\
\hline Rectum (R) & 80.0 & 2.2 & 0.7 & 3.0 & proctitis, necrosis, fistula, stenosis
\end{tabular}


Table 4. Mean values and variance of the different dose-volume parameter indices for the prostate target (PTV) and OARs using HIPO with and without (w/o) modulation restriction (MR)

\begin{tabular}{|c|c|c|c|c|c|}
\hline Organ & Parameter & HIPO & Mean value (\%) & Variance (\%) & $P$-value \\
\hline \multicolumn{6}{|c|}{ Targets } \\
\hline \multirow[t]{8}{*}{ PTV } & $\mathrm{D}_{90}$ & W MR & 102.5 & 2.3 & \multirow[t]{2}{*}{0.01} \\
\hline & & W/O MR & 103.1 & 3.2 & \\
\hline & $V_{100}$ & W MR & 92.2 & 1.5 & \multirow[t]{2}{*}{0.01} \\
\hline & & W/O MR & 92.7 & 2.1 & \\
\hline & $V_{150}$ & W MR & 30.4 & 4.3 & \multirow[t]{2}{*}{0.01} \\
\hline & & W/O MR & 29.2 & 5.4 & \\
\hline & $V_{200}$ & W MR & 8.7 & 1.3 & \multirow[t]{2}{*}{0.41} \\
\hline & & W/O MR & 8.6 & 1.4 & \\
\hline \multicolumn{6}{|c|}{ OARs } \\
\hline \multirow[t]{6}{*}{ Urethra } & $D_{1}$ & W MR & 117.4 & 3.2 & \multirow[t]{2}{*}{0.07} \\
\hline & & W/O MR & 118.1 & 2.3 & \\
\hline & $\mathrm{D}_{10}$ & W MR & 112.3 & 2.9 & \multirow[t]{2}{*}{0.23} \\
\hline & & W/O MR & 112.5 & 3.6 & \\
\hline & $D_{0.1 \mathrm{~cm}^{3}}$ & WMR & 114.3 & 2.8 & \multirow[t]{2}{*}{0.08} \\
\hline & & W/O MR & 114.6 & 3.1 & \\
\hline \multirow[t]{6}{*}{ Bladder } & $D_{10}$ & W MR & 45.4 & 15.8 & \multirow[t]{2}{*}{0.01} \\
\hline & & W/O MR & 45.9 & 17.2 & \\
\hline & $\mathrm{D}_{0.1 \mathrm{~cm}^{3}}$ & W MR & 73.1 & 7.3 & \multirow[t]{2}{*}{0.25} \\
\hline & & W/O MR & 73.4 & 3.9 & \\
\hline & $\mathrm{D}_{2 \mathrm{~cm}^{3}}$ & W MR & 55.6 & 22.5 & \multirow[t]{2}{*}{0.01} \\
\hline & & W/O MR & 56.2 & 22.9 & \\
\hline \multirow[t]{6}{*}{ Rectum } & $\mathrm{D}_{10}$ & W MR & 62.6 & 26.6 & \multirow[t]{2}{*}{0.04} \\
\hline & & W/O MR & 62.8 & 25.6 & \\
\hline & $D_{0.1 \mathrm{~cm}^{3}}$ & W MR & 76.6 & 2.1 & \multirow[t]{2}{*}{0.07} \\
\hline & & W/O MR & 76.4 & 1.6 & \\
\hline & $\mathrm{D}_{2 \mathrm{~cm}^{3}}$ & W MR & 62.7 & 18.3 & \multirow[t]{2}{*}{0.04} \\
\hline & & W/O MR & 62.9 & 17.3 & \\
\hline
\end{tabular}

functional subunits are damaged, whereas $\mathrm{s} \approx 1$ corresponds to a completely serial structure, which becomes nonfunctional when at least one functional subunit is damaged.

In tumors, the structural organization is assumed to be parallel since the eradication of all their clonogenic cells is required. Taking this feature into account the overall probability of benefit $P_{\mathrm{B}}$ is given by the expression:

$$
P_{\mathrm{B}}=\prod_{j=1}^{N_{\text {tumors }}} P_{\mathrm{B}}^{j}=\prod_{j=1}^{N_{\text {tumors }}}\left(\prod_{i=1}^{\mathrm{M}_{j}} \mathrm{P}^{j}\left(\mathrm{D}_{i}\right)^{\Delta v i}\right)
$$

where $P_{\mathrm{B}}^{j}$ is the probability of eradicating tumor $j$ and $N_{\text {tumors }}$ is the total number of tumors or targets involved in the clinical case. In Table 3, the dose-response parameters of the organs involved in this study are shown and are based on published data [29-33]. The $D_{50}$ and $\gamma$ parameters are derived from clinical materials and describe the shape of the dose-response curve, which subjects to uncertainties such as the inter-patient and intra-patient variation of radiosensitivity, setup uncertainties, accuracy of the radiobiological model, etc. [34,35]. The uncertainties that are associated with these parameters are of $5 \%$ for $D_{50}, 30 \%$ for $\gamma$ and $50 \%$ for $s[36,37]$. In parallel tissues (low $s$ value), the relative uncertainty of $s$ can be larger but this does not have a large impact on the calculations because it does not change the volume dependence of the tissue significantly. In this study, it was assumed that the patients are of average radiosensitivity.

The tissue response probabilities are calculated from the corresponding DVHs. Based on these data and applying them on the above models, the response probabilities of the individual tumors, $P_{\mathrm{B}}^{j}$, individual normal tissues, $P_{\mathrm{I}}^{j}$ as well as the total control, $\mathrm{P}_{\mathrm{B}}$ and total complication, $P_{1}$ probabilities were determined.

The biologically effective uniform dose, $\overline{\bar{D}}$, is defined as the dose that causes the same tumor control or normal tissue complication probability as the actual dose distribution delivered to the patient [16]. The general 
Table 5. Comparison of the COIN values, which were calculated with and without the inclusion of the organs at risk (OARs) for the 12 implants using HIPO with and without (w/o) modulation restriction (MR). The $p$ values of the paired $t$-test analysis are also presented

\begin{tabular}{|c|c|c|c|}
\hline \multicolumn{4}{|c|}{ COIN values including OARs } \\
\hline Case No & With MR & W/O MR $(M R=0)$ & $P$-value \\
\hline 1 & 0.869 & 0.862 & \multirow{12}{*}{0.0001} \\
\hline 2 & 0.884 & 0.862 & \\
\hline 3 & 0.866 & 0.863 & \\
\hline 4 & 0.867 & 0.862 & \\
\hline 5 & 0.896 & 0.887 & \\
\hline 6 & 0.841 & 0.823 & \\
\hline 7 & 0.876 & 0.863 & \\
\hline 8 & 0.881 & 0.875 & \\
\hline 9 & 0.869 & 0.864 & \\
\hline 10 & 0.836 & 0.829 & \\
\hline 11 & 0.881 & 0.863 & \\
\hline 12 & 0.841 & 0.832 & \\
\hline Mean \pm SD & $0.867 \pm 0.019$ & $0.857 \pm 0.019$ & \\
\hline \multicolumn{4}{|c|}{ COIN values without considering OARs } \\
\hline Case No & With MR & W/o MR $(M R=0)$ & $P$-value \\
\hline 1 & 0.881 & 0.873 & \multirow{12}{*}{0.003} \\
\hline 2 & 0.884 & 0.862 & \\
\hline 3 & 0.876 & 0.876 & \\
\hline 4 & 0.867 & 0.862 & \\
\hline 5 & 0.836 & 0.829 & \\
\hline 6 & 0.841 & 0.841 & \\
\hline 7 & 0.876 & 0.863 & \\
\hline 8 & 0.886 & 0.887 & \\
\hline 9 & 0.869 & 0.864 & \\
\hline 10 & 0.896 & 0.887 & \\
\hline 11 & 0.894 & 0.876 & \\
\hline 12 & 0.841 & 0.832 & \\
\hline Mean \pm SD & $0.870 \pm 0.021$ & $0.862 \pm 0.020$ & \\
\hline
\end{tabular}

expression of $\overline{\bar{D}}$ is derived numerically by the first part of the following equation, whereas for a tissue of uniform radiosensitivity, $\overline{\bar{D}}$ is given from the analytical formula of the second part:

$$
P(\vec{D}) \equiv P(\overline{\bar{D}}) \Rightarrow \overline{\bar{D}}=\frac{e \gamma-\ln (-\ln (P(\vec{D})))}{e \gamma-\ln (\ln 2)}
$$

where $\vec{D}$ denotes the 3-dimensional dose distribution delivered to the tissue and $P(\vec{D})$ is the response probability of the tissue. The second part of the equation has been derived using the Poisson model.

$P_{+}$is a scalar quantity, which expresses the probability of achieving tumor control without causing severe damage to normal tissues [17]. In this work, the probability of getting benefit from a treatment (total tumor control) was denoted by $P_{\mathrm{B}}$, whereas the probability for causing severe injury to normal tissues by $P_{\mathrm{J}}$. Using these quantities, $P_{+}$can be estimated from the following expression:

$$
P_{+}=P_{\mathrm{B}}-P_{\mathrm{B} \cap \mathrm{I}} \approx P_{\mathrm{B}}-P_{\mathrm{I}}
$$

In this study, to evaluate the effectiveness of the different treatment plans, conventional physical criteria like dose volume histograms, dose volume constraints, mean doses and dose variations in the target volumes and organs at risk were used together with the respective minimum and maximum doses and the radiobiological indices $\overline{\bar{D}}$ and $P_{+}$. 
Table 6. Summary of the dosimetric and radiobiological measures averaged over the 12 prostate cancer patients. The absolute values refer to the treatment plans optimized with HIPO utilizing the modulation restriction option (clinical plans), whereas the differences express the deviations of treatment plans optimized by HIPO without modulation restriction (MR) from the former ones regarding the applied HDR technique

\begin{tabular}{|c|c|c|c|c|c|c|c|c|c|c|c|c|}
\hline Structures & $P(\%)$ & $\Delta P(\%)$ & $D_{\text {mean }}$ & $\Delta D_{\text {mean }}$ & $C V(\%)$ & $\Delta C V$ & $\Delta \overline{\bar{D}}$ & $\overline{\bar{D}}$ & $D_{\max }$ & $\Delta D_{\max }$ & $D_{\min }$ & $\Delta D_{\min }$ \\
\hline CTV & 97.8 & 0.5 & 48.4 & -0.3 & 30.5 & 0.1 & 32.9 & 0.3 & 136.6 & 0.0 & 23.5 & 0.0 \\
\hline Urethra & 3.8 & 1.0 & 33.0 & 0.4 & 18.2 & -1.2 & 34.2 & 0.2 & 41.4 & 1.4 & 11.0 & 0.0 \\
\hline Bladder & 0.0 & 0.0 & 9.2 & 0.1 & 46.5 & 0.0 & 22.3 & 0.0 & 27.6 & 0.0 & 2.8 & 0.0 \\
\hline Rectum & 0.02 & 0.00 & 14.4 & 0.2 & 33.3 & -0.6 & 22.8 & 0.0 & 27.6 & 0.0 & 2.8 & 0.0 \\
\hline \multicolumn{2}{|c|}{ Dose prescription } & \multicolumn{5}{|c|}{ Clinical } & & \multicolumn{5}{|c|}{ Optimum $P_{+}$} \\
\hline \multicolumn{2}{|c|}{ HIPO Optimization } & \multicolumn{2}{|c|}{ with $M R$} & \multicolumn{3}{|c|}{ without MR } & & \multicolumn{3}{|c|}{ with MR } & \multicolumn{2}{|r|}{ without MR } \\
\hline \multicolumn{2}{|l|}{$P_{+} / \Delta P_{+}(\%)$} & \multicolumn{2}{|c|}{94.0} & \multicolumn{3}{|c|}{-0.5} & & \multicolumn{3}{|c|}{95.2} & \multicolumn{2}{|r|}{0.2} \\
\hline \multicolumn{2}{|l|}{$P_{\mathrm{B}} / \Delta P_{\mathrm{B}}(\%)$} & \multicolumn{2}{|c|}{97.8} & \multicolumn{3}{|c|}{0.5} & & \multicolumn{3}{|c|}{96.3} & \multicolumn{2}{|r|}{-0.2} \\
\hline \multicolumn{2}{|l|}{$P_{r} / \Delta P_{r}(\%)$} & \multicolumn{2}{|c|}{3.8} & \multicolumn{3}{|c|}{1.0} & & \multicolumn{3}{|c|}{1.1} & \multicolumn{2}{|r|}{-0.4} \\
\hline \multicolumn{2}{|l|}{$\overline{\bar{D}}_{\mathrm{B}} / \Delta \overline{\bar{D}}_{\mathrm{B}}(\mathrm{Gy})$} & \multicolumn{2}{|c|}{32.9} & \multicolumn{3}{|c|}{0.3} & & \multicolumn{3}{|c|}{32.2} & & -0.1 \\
\hline
\end{tabular}

\section{Results}

The results of the differences observed in the different dosimetric and radiobiological indices for the prostate and OARs, respectively are listed in Tables 4-6. It is observed that the values of the parameter $\mathrm{D}_{90}$ for the prostate were significantly lower $(p=0.01)$ when the MR was used than without using the MR. The same behavior applies for the $\mathrm{V}_{100}$ of prostate although the average absolute difference of the $D_{90}$ and $V_{100}$ values were always less than $1 \%$. The opposite happens for the $V_{150}$ and the $V_{200}$ parameters of prostate where the values were greater when the MR was used ( $p=0.01$ and $p=0.41$, respectively). For urethra, the parameters $\mathrm{D}_{1}, \mathrm{D}_{10}$ and $\mathrm{D}_{0.1 \mathrm{~cm}^{3}}$ were reduced when using MR but their difference was not significant. For bladder and rectum, modulation restriction resulted in a significant reduction of the $D_{10}$ parameter values with $p=0.01$ and $p=0.04$, respectively. The observed differences in $\mathrm{D}_{0.1 \mathrm{~cm}^{3}}$ values are for both OARs not significant. The $\mathrm{D}_{2 \mathrm{~cm}} \mathrm{~cm}^{3}$ parameter value, for both rectum and bladder, was significantly lower when using MR $(p=0.04$ and 0.01 , respectively).

Table 5 compares the results of COIN for the two different plan categories. In the case of COIN considering only the target the observed differences (higher COIN values when MR is used) were significant $(p=0.003)$. When OARs were also considered the COIN values were again significantly higher $(p=0.0001)$ when MR was used. The dose limit values for the three OARs for calculating COIN including OARs according to equation (3) were considered to be the corresponding $\mathrm{D}_{0.1 \mathrm{~cm}^{3}}$ values as listed in Table 1 .

In this study, the effectiveness of treatment plans produced by HIPO with and without MR was compared by evaluating their physical and radiobiological characteristics. Figure 3 shows comparison of these two dose distribution sets in terms of differential DVHs. In Fig. 4, the average DVHs of the prostate, urethra, bladder and rectum are presented, whereas in Fig. 5, the average doseresponse curves of the PTV and individual OARs are shown for different prescription doses. This means that in every prescription dose examined, the average response probabilities have been calculated and plotted for every tissue. In Fig. 5, the average dose-response curves of total tumor control and total normal tissue complication probabilities are presented together with the average $P_{+}$ curve. In this diagram, the dose-response curves were normalized to the $\overline{\bar{D}}_{\mathrm{B}}$, which forces the response curves of the PTV $\left(P_{\mathrm{B}}\right)$ of the different treatment plans to coincide. In these diagrams the same dose distribution was kept at all dose levels and the curves show how tissue responses change with the dose prescription. The normalization using $\overline{\bar{D}}_{\mathrm{B}}$ allows the inter-comparison of the different modalities on the same basis and gives emphasis to the therapeutic window, which characterizes each treatment plan.

In Table 6, a quantitative summary of the dosimetric and radiobiological results of the different dose distributions is presented. For the HDR optimization with MR and at the clinical dose prescription the $P_{+}$value was $94.0 \%$ and the biologically effective uniform dose to the PTV, $\overline{\bar{D}}_{\mathrm{B}}$ was $32.9 \mathrm{~Gy}$. The total control probability, $P_{B}$ is $97.8 \%$ and the total complication probability, $P_{I}$ is $3.8 \%$, which mainly stems from the response probability of urethra $(3.8 \%)$. Similarly, for the HDR optimization without MR, the $P_{+}$value was $93.5 \%$ for a $\overline{\bar{D}}_{\mathrm{B}}$ of $33.2 \mathrm{~Gy}$. The value of $P_{B}$ was $98.3 \%$ and that of PI was $4.8 \%$, which was almost equal to the response probability of the urethra. However, if we optimize the dose level of the dose distributions in order to maximize the complication-free tumor control then for the HIPO with MR plans, the $P_{+}$ value becomes $95.2 \%$ for a $\overline{\bar{D}}_{\mathrm{B}}$ of $32.2 \mathrm{~Gy}$ having $P_{\mathrm{B}}=96.3 \%$ and $P_{\mathrm{I}}=1.1 \%$. Respectively, for the HIPO without MR plans, the $P_{+}$value becomes $95.4 \%$ for a $\overline{\bar{D}}_{\mathrm{B}}$ of $32.1 \mathrm{~Gy}$. The corresponding average $P_{\mathrm{B}}$ was $96.1 \%$, whereas the average $P_{\mathrm{I}}$ is $0.7 \%$.

The diagram of Fig. 4 illustrates the average DVHs of the two series of dose distributions examined. Based on the DVHs and the results shown in Table 6, the HIPO optimization without MR has higher variance coefficient, 

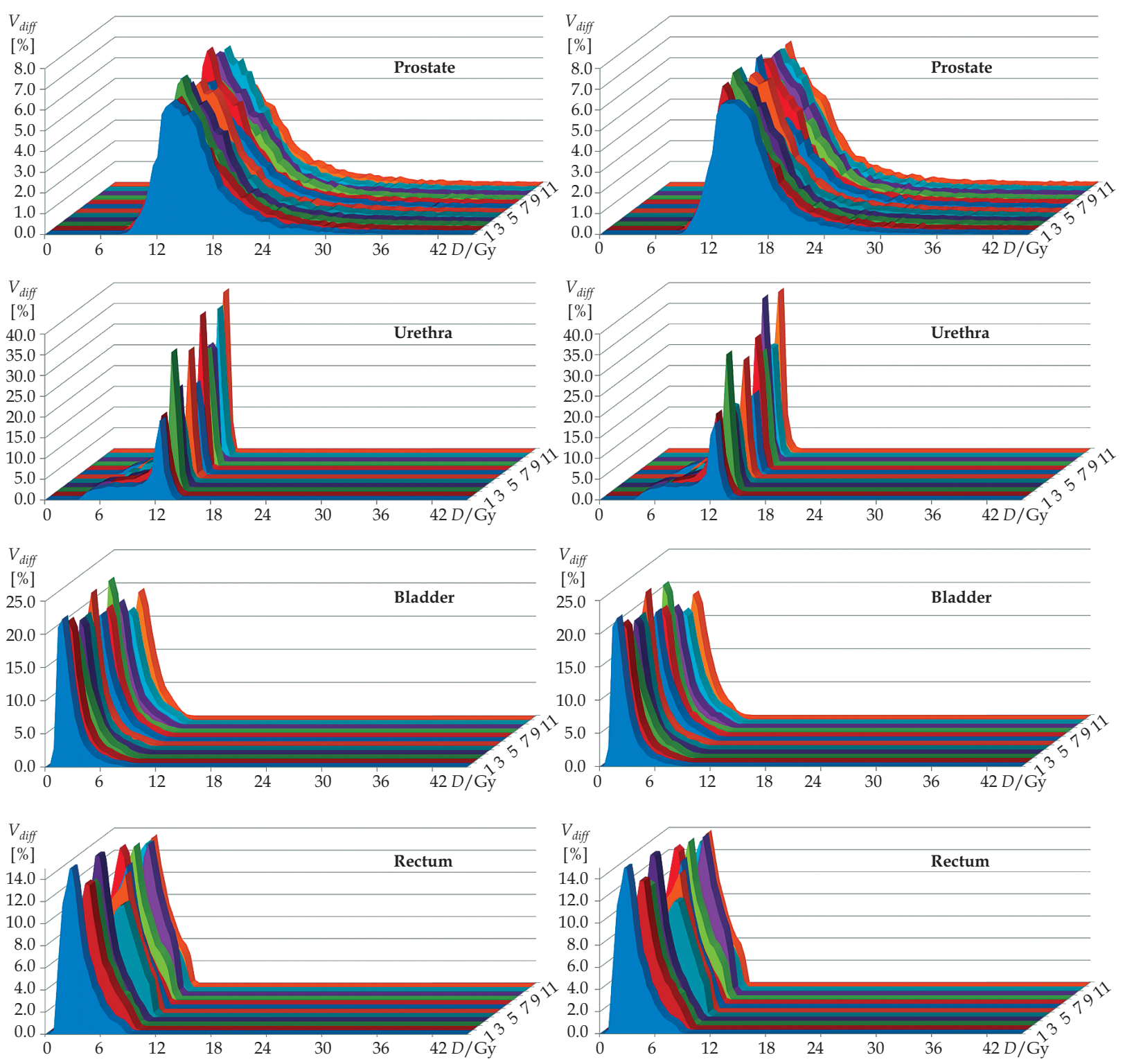

Fig. 3. The differential DVHs of prostate, urethra, bladder and rectum derived from the treatment plans with (left) and without (right) modulation restriction. The prescription dose of the fraction is $11.5 \mathrm{~Gy}(100 \%)$

CV, (meaning larger dose inhomogeneity) inside the PTV than the HIPO optimization with MR. However, although the average mean dose in the PTV was lower in the HIPO without MR plans by 0.3 Gy (48.4 Gy vs. $48.1 \mathrm{~Gy}$ ), the corresponding control probability was higher by $0.5 \%$ (97.8\% vs. $98.3 \%$ ) compared to HIPO with MR (Table 6). Regarding the organs at risk, the HIPO optimization without MR plans delivers higher maximum doses in urethra than the HIPO optimization with MR (difference of $1.4 \mathrm{~Gy})$.

\section{Discussion}

The present analysis shows that the HIPO HDR optimization with modulation restriction appears to be slightly more effective in treating the prostate than the
HIPO HDR optimization without modulation restriction. The tumor and normal tissue response probabilities were estimated using radiobiological models and published parameter sets. From the diagrams of Fig. 5 it is apparent that urethra is the dose limiting tissue for both optimization approaches. This is because of the high mean doses delivered to this tissue by the corresponding treatment plans. In the diagrams of Fig. 6 , it is shown that the expected $P_{+}$value for the HDR optimization with modulation restriction is higher than the HDR optimization without modulation restriction.

The differences observed on the DVH comparisons between the two optimization methods are not always reflected in the radiobiological evaluation, which uses the dose-response relations of the tissues involved. This is because the way a certain dose distribution affects an organ 


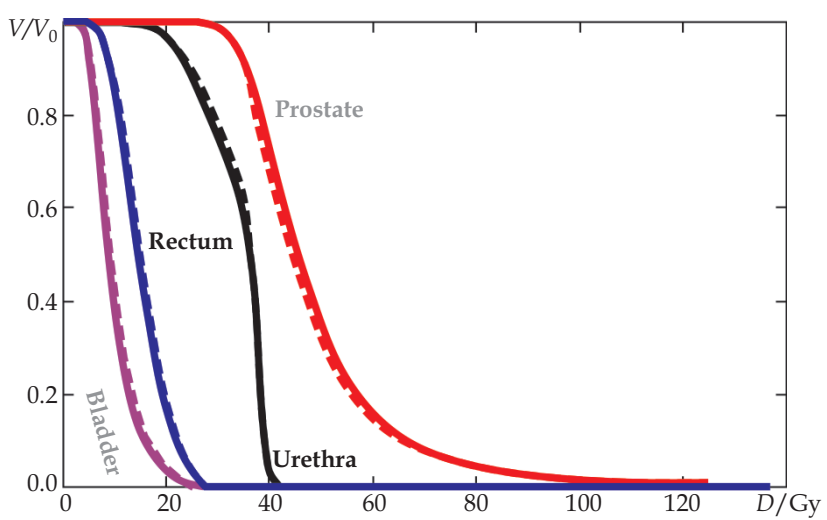

Fig. 4. The average DVHs of the PTV (prostate gland, red), urethra (black), bladder (pink) and rectum (blue) are presented for the HDR treatment plans, which were optimized with (solid) and without (dashed) modulation restriction, separately. Here, the total dose of 34.5 Gy delivered by three fractions of $11.5 \mathrm{~Gy}$ is considered to be the total prescription dose $(100 \%)$

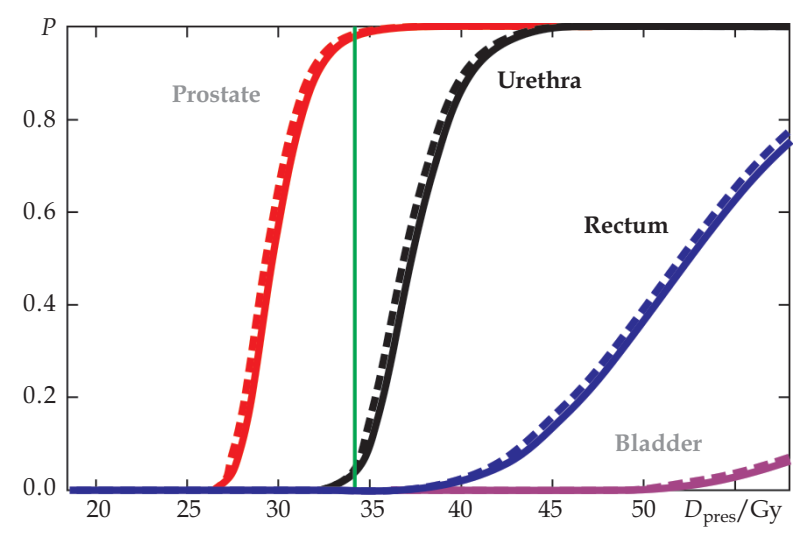

Fig. 5. The average dose-response curves of the PTV (red), urethra (black), bladder (pink) and rectum (blue) are presented for the HDR treatment plans, which were optimized with (solid) and without (dashed) modulation restriction (MR), separately regarding different prescription doses. The vertical line indicates the clinical prescribed dose level of the dose distributions produced by HIPO with and without MR. The probability values are calculated using equations (4) and (5) and are based on the DVHs of figure 4. Here the total dose of 34.5 Gy delivered by three fractions of $11.5 \mathrm{~Gy}$ is considered to be the total prescription dose (100\%)

depends on its radiobiological characteristics Observing the diagrams in Fig. 6, it is apparent that in the qualitative treatment plans the curve of $P_{+}$becomes higher and the width of the therapeutic window broadens, since the response curves of the tumors and the involved normal tissues move away from each other. By using the biologically effective uniform dose, $\overline{\bar{D}}$ concept, a number of plan trials can be compared by normalizing their dose distributions to a common prescription point $(\bar{D})$ and then plotting the tissue response probability curves vs. $\overline{\bar{D}}$.

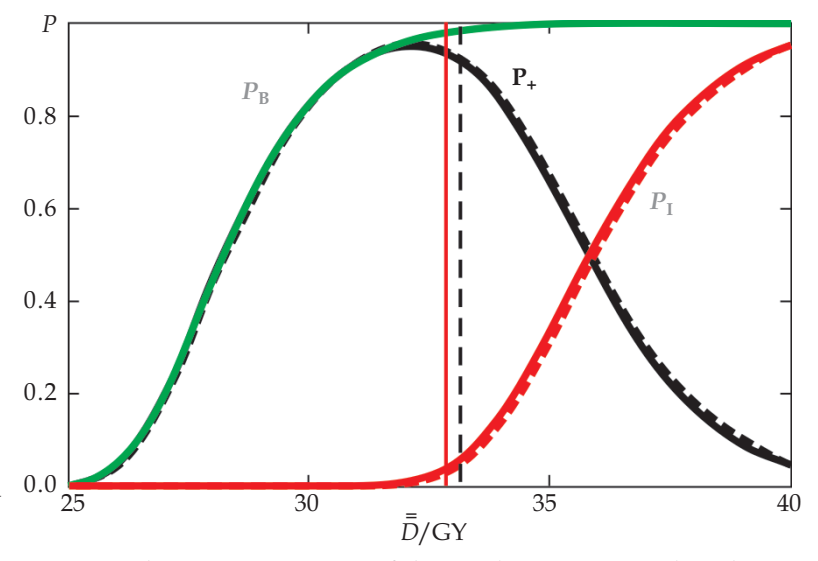

Fig. 6. The average curves of the total tumor control probability, $P_{B}$ (green), total normal tissue complication probability, $P_{I}$ (red) and complication-free tumor control probability, $P_{+}$(black) are presented for the HDR treatment plans, which were optimized with (solid) and without (dashed) modulation restriction (MR), separately regarding different radiobiological prescription doses. The solid and dashed vertical lines indicate the radiobiological dose levels of the dose distributions produced by HIPO with and without MR, respectively. The probability values are calculated using equations (4)-(7) and are based on the DVHs of Fig. 4. Here, the total dose of 34.5 Gy delivered by three fractions of $11.5 \mathrm{~Gy}$ is considered to be the total prescription dose $(100 \%)$

According to the DVHs shown in the diagrams of Fig. 4 and results of Table 6, the following conclusions can be made. First, in the HIPO optimizations with and without MR, the DVHs of the prostate are characterized by very similar dose gradients around the PTV. Furthermore, the HIPO optimization with MR delivers lower doses to the OARs compared to the HIPO optimization without MR case.

Generally, the goodness or effectiveness of optimized treatment plans can be assessed by different criteria. The dosimetric quantities used in most studies have an indirect relation to the clinical outcome. A comparison using such quantities does not always depict a corresponding relation in the clinic. In the present study, dose-response measures are used instead to show the clinical impact of the dosimetric results. This is because these dose-response measures have been derived from real clinical data and refer directly to the final treatment outcome.

The conclusions derived by the dosimetric comparisons (Tables 4 and 5) are supported to a large extent by the results of the radiobiological evaluation and comparison (Table 6). However, it seems that the optimal way to optimize HDR treatment plans is to incorporate radiobiological measures in inverse planning, which will refer to the expected clinical outcome. That is because the optimization of the dose gradient from the tumor towards a certain organ at risk depends very much on the radiobiological characteristics of that organ and especially on its volume effect dependence, which expresses the capability of that organ to sustain high doses to a fraction of its volume. 
The effectiveness of a given dose distribution is evaluated by the comparison of its advantages in terms of tumor control against its disadvantages regarding normal tissue complications. The original definition of $P_{+}$does not use different weights for the targets and OARs as well as different weights for the different normal tissues. In clinical practice, there are not different weighting factors that are applied but there are risk thresholds (usually 5-10\%) for every organ at risk, which should not be exceeded. So, in order to classify the different treatment plans one can select in the diagrams of Figs. 5 and 6 the dose level that satisfies the demands imposed by the normal tissues risk thresholds and associate them with the expected tumor control rate at this dose level. By performing this analysis for the prostate cancer case, we can observe that at the clinically defined dose levels of the HDR optimization with and without MR schemes, the respective expected tumor control rates are $97.8 \%$ and $98.3 \%$, when the expected normal tissue complication probabilities are below $5 \%$ (3.8\% and $4.8 \%$ for urethra, respectively) for each of the critical organs examined.

Regarding the target volumes, the dose non-uniformity in the HDR optimization with MR is slightly lower than in the HDR optimization without MR when looking to the coefficient of variance (Table 6). This is because the HDR optimization with MR and HDR optimization without MR algorithms optimize this dose fall-off around the target, differently. In radiobiological terms there may exist nonuniform dose distributions, which may be as effective as their equivalent uniform dose distribution. In this sense, a radiobiologically based optimization algorithm would take advantage of the higher number of degrees-offreedom provided by the radiobiological measures and find dose distributions of smoother non-uniformity that irradiate the target as effectively as the dose distribution without MR and at the same time optimize the dose falloff towards the organs at risk. This is because the radiobiologically based HDR optimization would take into account the volume effect of all the involved organs at risk in the proximity of the target and optimize the dose falloff accordingly. The radiobiological measures used to evaluate the different dose distributions support this analysis. It has to be mentioned that the HDR optimization without MR is characterized by a higher number of degrees-of-freedom than the HDR optimization with MR and by using a more biologically relevant dose constraint for the normal tissue stroma it could lead to better results than the latter method. However, the large hot spots produced in the target volume by this method would increase the risk for secondary cancer [38]. Consequently, by deteriorating physical dose conformation, the HDR optimization with MR provides slightly better biological conformation.

According to previous experience in determining doseresponse parameters for various tissues, it has been observed that different radiobiological models behave better in different cases. Most of the existing models need to be further expanded in order to describe accurately further underlying biological mechanisms. However, even in their present form their accuracy can be quite good as long as they use accurate parameter values. For this purpose, these values must have been determined from well-designed studies using accurately delivered 3D dose distributions and well-defined and measured follow-up data considering every individual patient. It seems that the accuracy of the model parameters and their compatibility with the clinical case under study are the most important factors to achieve a good accuracy in the radiobiological evaluation of a treatment plan. In general, the use of DVHs instead of using 3D dose distributions is based on the fact that most of the existing radiobiological models do not make any use of the spatial information. In other words, these models do not distinguish the organ-cells or voxels according to their positions but according to the dose they receive. By using more advanced imaging information it will soon be possible to take into account more spatial-related information and then the use of 3D dose matrices will be a necessity.

\section{Conclusions}

In this study, twelve prostate cancer patients were employed for evaluating the clinical effectiveness of the treatment plans produced by the HIPO optimization algorithm using the Modulation Restriction functionality. The evaluation was performed using both physical and radiobiological criteria. This evaluation shows that the HDR optimization with MR can introduce a minor improvement in the effectiveness of the produced dose distribution compared to the HDR optimization without modulation restriction. The likelihood to accomplish a good treatment result can be increased by the use of therapeutic indices such as $P_{+}$and $\overline{\bar{D}}$, which can be used as figures of merit for a treatment. The simultaneous presentation of the radiobiological evaluation together with the physical data shows their complementary relation in analyzing a dose plan. The use of radiobiological parameters is necessary if a clinically relevant quantification of a plan is needed.

\section{References}

1. Mould RF, Martinez AA, Orton CG. Brachytherapy HDR and LDR. AIP, New York 1989.

2. Baltas D, Zamboglou N. 2D and 3D Planning in Brachytherapy. In: Schlegel W, Bortfeld T, Grosu AL. New Technologies in Radiation Oncology. Springer, New York 2006.

3. Lahanas M, Baltas D, Giannouli S. Global convergence analysis of fast multiobjective gradient based dose optimization algorithms for high-dose-rate brachytherapy. Phys Med Biol 2003; 48: 599-617.

4. Karabis A, Belotti P, Baltas D. Optimization of Catheter Position and Dwell Time in Prostate HDR Brachytherapy using HIPO and Linear Programming, World Congress on Medical Physics and Biomedical Engineering, September 7-12, 2009, Munich, Germany, IFMBE Proceedings 25/I. Springer, 2009: 612-615.

5. Lessard E, Pouliot J. Inverse planning anatomy-based dose optimization for HDR brachytherapy of the prostate using fast simulated annealing algorithm and dedicated objective function. Med Phys 2001; 28: 773-779.

6. Lahanas M, Baltas D, Zamboglou N. Anatomy-based threedimensional dose optimization in brachytherapy using multiobjective genetic algorithms. Med Phys 1999; 26: 1904-1918.

7. Alterovitz R, Lessard E, Pouliot J et al. Optimization of HDR brachytherapy dose distributions using linear programming with penalty costs. Med Phys 2006; 33: 4012-4019. 
8. Oncentra Prostate ${ }^{\mathrm{TM}}$, Nucletron B.V. , Waardgelder 1, TH 3905 Veenendaal, The Netherlands.

9. BrachyVision Treatent Planning ${ }^{\text {TM }}$, Varian Medical Systems.

10. Swamidas JV, Kirisits C, Mahantshetty U et al. Loading pattern calculated by inverse optimization vs traditional dosimetry systems of intracavitary brachytherapy of cervical cancer - a dosimetric study. Brachytherapy 2009; 8: 127.

11. Aydogan B, Mundt AJ, Smith BD et al. A dosimetric analysis of intensity-modulated radiation therapy (IMRT) as an alternative to adjuvant high-dose-rate (HDR) brachytherapy in early endometrial cancer patients. Int J Radiat Oncol Biol Phys 2006; 65: 266-273.

12. Karabis A, Giannouli S, Baltas D. "HIPO: A hybrid inverse treatment planning optimization algorithm in HDR brachytherapy", presented in 8th Biennial ESTRO Meeting Physics and Radiation Technology for Clinical Radiotherapy, Lisboa, 24-29/09/2005 (published in Radiotherapy E Oncology, Vol. 76, Supplement 2, Sept. 2005, p. S29).

13. Mavroidis P, Komisopoulos G, Lind BK et al. Interpretation of the dosimetric results of three uniformity regularization methods in terms of expected treatment outcome. Med Phys 2008 35: 5009-5018.

14. Baltas D, Kolotas C, Geramani K et al. A Conformal Index (COIN) to evaluate implant quality and dose specification in brachytherapy. Int J Radiat Oncol Biol Phys 1998; 40: 515-524.

15. Milickovic N, Lahanas M, Papagiannopoulou M et al. Multiobjective anatomy-based dose optimization for HDRbrachytherapy with constraint free deterministic algorithms. Phys Med Biol 2002; 47: 2263.

16. Mavroidis P, Lind BK, Brahme A. Biologically effective uniform dose for specification, report and comparison of dose response relations and treatment plans. Phys Med Biol 2001; 46: 2607-2630.

17. Källman P, Lind BK, Brahme A. An algorithm for maximizing the probability of complication free tumor control in radiation therapy. Phys Med Biol 1992; 37: 871-890.

18. Kovac's G, Poetter R, Loch $\mathrm{T}$ et al. GEC/ESTRO-EAU recommendations on temporary brachytherapy using stepping sources for localised prostate cancer. Radiother Oncol 2005; 74: 137-148.

19. Baltas D, Zampoglou N. 2D and 3D Planning in Brachytherapy. In: Bortfeld T, Grosu A-L, Schlegel W. New Technologies in Radiation Oncology. Springer, Berlin-Heidelberg 2006.

20. Nag S, Beyer D, Friedland J et al. American Brachytherapy Society (ABS) Recommendations for Transperineal Permanent Brachytherapy of Prostate Cancer. Int J Radiat Oncol Biol Phys 1999; 44: 789-799.

21. Hsu I-C, Yamada Y, Vigneault E, Pouliot J. 2008; available at: http://www.americanbrachytherapy.org/.

22. Pötter R, Van Limbergen E, Wambersie A. Reporting in Brachytherapy: Dose and Volume specification. THE GEC ESTRO Handbook of Brachytherapy. ESTRO, Brussels 2002.

23. Drzymala RE, Mohan R, Brewster L et al. Dose-Volume Histograms. Int J Radiat Oncol Biol 1991; 21: 71-78.

24. Marks LB, Sherouse GW, Munley MT et al. Incorporation of functional status into dose-volume analysis. Med Phys 1999; 26 : 196-199.

25. Cheng CW, Das IJ. Treatment plan evaluation using dose-volume histogram (DVH) and spatial dose-volume histogram (zDVH). Int J Radiat Oncol Biol Phys 1999; 43: 1143-1150.

26. Inoue T. Current status and perspectives of brachytherapy. Int J Clin Oncol 2009; 14: 1 [Epub 2009 Feb 20.].

27. Källman P, Ågren A-K, Brahme A. Tumor and normal tissue responses to fractionated non uniform dose delivery. Int J Radiat Biol 1992; 62: 249-62.
28. Ågren-Cronqvist A-K, Brahme A, Turesson I. Optimization of uncomplicated control for head and neck tumors. Int J Radiat Oncol Biol Phys 1990; 19: 1077-1085.

29. Emami B, Lyman J, Brown A et al. Tolerance of normal tissue to therapeutic irradiation. Int J Radiat Oncol Biol Phys 1991; 21: 109-122.

30. Ågren A-K. Quantification of the response of heterogeneous tumors and organized normal tissues to fractionated radiotherapy. Ph.D. Thesis. Stockholm University, Stockholm 1995.

31. Wang JZ, Guerrero M, Allen X. How low is the $\alpha / \beta$ ratio for prostate cancer? Int J Radiat Oncol Biol Phys 2003; 55: 194-203.

32. Wang JC, Li XA. Impact of tumour repopulation on radiotherapy planning. Int J Radiat Oncol Biol Phys 2005; 61: 220-227.

33. http://www.gla.ac.uk/sums/users/jdbmcdonald/PrePost_TTest/pairedt1.html.

34. Mavroidis P, Ferreira BC, Shi C et al. Treatment plan comparison between helical tomotherapy and MLC-based IMRT using radiobiological measures. Phys Med Biol 2007; 52: 3817-3836.

35. Ferreira BC, Mavroidis P, Adamus-Górka M et al. The impact of different dose-response parameters on biologically optimized IMRT in breast cancer. Phys Med Biol 2008; 53: 2733-2752.

36. Mavroidis P, Laurell G, Kraepelien T et al. Determination and clinical verification of dose-response parameters for esophageal stricture from head and neck radiotherapy. Acta Oncol 2003; 42: 865-881.

37. Mavroidis P, al-Abany M, Helgason AR et al. Dose-response relations for anal sphincter regarding faecal leakage and blood or phlegm in stools after radiotherapy for prostate cancer. Strahlenther Onkol 2005; 181: 293-306.

38. Schneider U, Lomax A, Pemler P et al. The impact of IMRT and proton radiotherapy on secondary cancer incidence. Strahlenther Onkol 2006; 182: 647-652. 